\title{
Estruturação documental do serviço de farmácia clínica em uma unidade de terapia intensiva de um hospital público de grande porte
}

Documentary structuring of the clinical pharmacy service in an intensive treatment center in a large public hospital Estructuración documental del servicio de farmacia clínica en una unidad de cuidados intensivos de un gran hospital público

\begin{abstract}
RESUMO
Objetivo: Descrever a estruturação e implementação de farmácia clínica na unidade de terapia intensiva. Métodos: Trata-se de estudo descritivo, cuja metodologia foi elaboração de fluxograma de atividades desenvolvidas pelo farmacêutico e formulários para facilitar acompanhamento farmacoterapêutico. Para confecção dos documentos foi realizada uma pesquisa bibliográfica e consulta a outros formulários de acompanhamento clínico da própria instituição e de outros serviços de farmácia clínica. Resultados: Foram criados documentos estruturantes: fluxograma do serviço de farmácia; formulário de acompanhamento farmacoterapêutico; check-list de avaliação da prescrição e check-list de acompanhamento farmacoterapêutico; modelo de notificação de intervenção farmacêutica; e, banco de dados para registro das intervenções a serem realizadas. Esses documentos contam com informações importantes para o acompanhamento diário do paciente. Conclusão: 0 método proposto auxilia no desenvolvimento de atividades clínicas, promovendo integração do farmacêutico à equipe multiprofissional, cuidado integral do paciente e segurança. Adaptação a formulário próprio garante a equipe conduzir acompanhamento de personalizado.
\end{abstract}

DESCRITORES: Serviço de Farmácia Hospitalar; Tratamento Farmacológico; Unidades de Terapia Intensiva; Segurança do Paciente.

\section{ABSTRACT}

Objective: To describe the structuring and implementation of clinical pharmacy in the intensive care unit. Methods: This is a descriptive study, whose methodology was the elaboration of a flowchart of activities developed by the pharmacist and forms to facilitate pharmacotherapeutic follow-up. To make the documents, a bibliographic search and other forms of clinical follow-up from the institution and other services of clinical pharmacy were carried out. Results: Structural documents were created: flowchart of the pharmacy service; pharmacotherapeutic follow-up form; prescription evaluation checklist and pharmacotherapeutic follow-up checklist; pharmaceutical intervention notification model; and, a database to record the interventions to be performed. These documents have important information for the daily monitoring of the patient. Conclusion: The proposed method assists in the development of clinical activities, promoting integration of the pharmacist to the multidisciplinary team, comprehensive patient care and safety. Adaptation to a specific form guarantees the team to conduct personalized monitoring.

DESCRIPTORS: Hospital Pharmacy Service; Drug Therapy; Intensive Care Units; Patient Safety.

\section{RESUMEN}

Objetivo: Describir la estructuración e implementación de la farmacia clínica en la unidad de cuidados intensivos. Métodos: Se trata de un estudio descriptivo, cuya metodología fue la elaboración de un diagrama de flujo de actividades desarrolladas por el farmacéutico y formularios para facilitar el seguimiento farmacoterapéutico. Para la confección de los documentos se realizó una búsqueda bibliográfica y otras formas de seguimiento clínico desde la institución y otros servicios de farmacia clínica. Resultados: Se crearon documentos estructurales: diagrama de flujo del servicio de farmacia; formulario de seguimiento farmacoterapéutico; lista de verificación de evaluación de prescripciones y lista de verificación de seguimiento farmacoterapéutico; modelo de notificación de intervenciones farmacéuticas; $y$, una base de datos para registrar las intervenciones a realizar. Estos documentos contienen información importante para el seguimiento diario del paciente. Conclusión: El método propuesto ayuda al desarrollo de las actividades clínicas, promoviendo la integración del farmacéutico al equipo multidisciplinario, la atención integral del paciente y la seguridad. La adaptación a una forma específica garantiza al equipo realizar un seguimiento personalizado.

DESCRIPTORES: Servicio de Farmacia en Hospital; Quimioterapia; Unidades de Cuidados Intensivos; Seguridad del Paciente.

RECEBIDO EM: 15/12/2020 APROVADO EM: 11/01/2021 


\section{Thais Silva Christiani}

Farmacêutica Especialista em Farmácia Hospitalar. Farmacêutica Residente em Farmácia Hospitalar, Universidade Federal Fluminense, Rio de Janeiro.

ORCID: 0000-0003-1491-8237

\section{Gabrielle da Costa Rocha}

Farmacêutica Especialista em Farmácia Hospitalar. Farmacêutica Residente em Farmácia Hospitalar, Universidade Federal Fluminense, Rio de Janeiro.

ORCID: 0000-0001-9988-9249

\section{Thaísa Amorim Nogueira}

Farmacêutica Doutora em Ciências Aplicadas a Produtos para Saúde. Professora do Curso de Farmácia, Universidade Federal do Rio de Janeiro Campus Macaé, Rio de Janeiro.

ORCID: 0000-0003-0527-4417

\section{INTRODUÇÃO}

A tualmente na farmácia hospitalar o farmacêutico envolve-se nas etapas de seleção, aquisição, armazenamento, controle e distribuição de medicamentos. Ademais, a contribuição recente desse profissional na farmácia clínica tem proporcionado maior efetividade no processo de uso racional dos medicamentos, maximizando a terapia e diminuindo os riscos e os custos ${ }^{1,2,3,4}$.

A resolução do Conselho Federal de Farmácia (CFF) no 585 , de 29 de agosto de 2013, define farmácia clínica como uma "área voltada à ciência e prática do uso racional de medicamentos, na qual os farmacêuticos prestam cuidado ao paciente, de forma a otimizar a farmacoterapia, promover saúde e bem-estar e prevenir doenças" " ${ }^{5}$. Neste cenário, o farmacêutico na farmácia clínica realiza, sobretudo, o acompanhamento farmacoterapêutico que envolve uma sequência racional de ações que buscam monitorar a terapia medicamentosa e avaliar o cumprimento dos objetivos terapêuticos ao quais ela se propõe, visando a efetividade e segurança ${ }^{4,6}$.

Para a implantação de uma farmácia clínica alguns pré-requisitos devem ser atendidos, a saber: existência de sistema de distribuição de medicamentos seguro, tempo para a prática clínica farmacêutica, apoio de gestores, inserção do farmacêutico na equipe multiprofissional, entre outros fatores ${ }^{1}$.

O cenário de atendimento de unidades de tratamento intensivo por vezes se torna complexo aos profissinais pelo estado crítico do paciente. Desta forma, a equipe multidisciplinar trabalha com processos por vezes mal desenhados, utilização desnecessária de material e medicamentos, limitação de espaço para estoque no setor, alta carga de trabalho para equipe de enfermagem, dificultando a implementação e manutenção de treinamentos e educação continuada. Assim esse sistema se retroalimenta com permanência de processos mal elaborados e por vezes inseguros aos pacientes.?

A equipe de saúde por vezes em trabalho

\section{Atualmente na \\ farmácia hospitalar \\ o farmacêutico}

envolve-se nas

etapas de seleção,

aquisição,

armazenamento,

controle e distribuição

de medicamentos. conjunto promoverá assistência segura e completa ao paciente em estado crítico. Alguns achados em terapias medicamentosas podem ser otimizados com a informação adequada a médicos e enfermeiros sobre o manejo dos medicamentos. Dose, posologia, via de administração adequados para terapia podem ser informações relevantes a equipe médica durante a confecção da prescrição. Assim como intervalos para administração do medicamento, diluentes adequados, volumes para reconstituição, diluição; e tempo de infusão são primordiais para boa conduta da enfermagem. ${ }^{89}$

O farmacêutico deve estar integrado à equipe multiprofissional, promovendo o acompanhamento diário dos pacientes e buscando contribuir na qualidade da assistência 6 , tendo como objetivo a segurança e utilização correta da farmacoterapia, podendo este profissional identificar e resolver os problemas relacionados a medicamentos $4,7,8,9$.

É importante que haja uma comunicação eficaz e eficiente entre os profissionais de saúde, sendo assim, o farmacêutico deve documentar os cuidados prestados e as intervenções realizadas, uma vez que essa informação é vital para a progressão do cuidado do paciente ${ }^{10}$. Alguns autores recomendam que essa documentação seja registrada no prontuário, uma vez que esse documento é de acesso comum a todos os profissionais de saúde ${ }^{3,11}$.

Recentemente aprovada, a Resolução n ${ }^{\circ} 675$ do CFF, de 31 de outubro de 2019, regulamenta as atribuições do farmacêutico clínico em unidade de terapia intensiva (UTI) ${ }^{12}$. A UTI destina-se ao atendimento de pacientes críticos que comumente requerem cuidados intensivos, com uso de diversos 
medicamentos, por vezes de administração endovenosa, o que aumenta significativamente os riscos de interações e incompatibilidades medicamentosas ${ }^{4,13}$. Esses pacientes possuem alto risco de apresentarem erros relacionados a medicamentos, devido à natureza crítica da doença, à polifarmácia, à utilização de medicamentos de alto risco e frequente alteração na farmacoterapia ${ }^{14,15}$.

Tendo em vista a complexidade dos cuidados que os pacientes críticos requerem, diversos autores recomendam a presença do farmacêutico na UTI ${ }^{6,14,16,17}$. Este farmacêutico deve ter habilidade para atuar em todo processo relacionado ao medicamento $^{14}$. Considerando ainda que a assistência ao paciente crítico tem um papel decisivo em sua chance de sobrevida ${ }^{18}$, torna-se fundamental o acompanhamento da farmacoterapia. Desta forma este artigo descreve um modelo para a estruturação documental de um serviço de Farmácia Clínica na UTI de um Hospital Federal de grande porte localizado na cidade do Rio de Janeiro.

\section{MÉTODOS}

Trata-se de um estudo documental descritivo desenvolvido para estruturação de formulários a serem aplicados na impantação de farmácia clínica da UTI de um hospital federal de grande porte e alta complexidade localizado na cidade do Rio de Janeiro, com cerca de 400 leitos, distribuídos em 16 unidades de internação, incluindo uma UTI com 10 leitos.

Inicialmente foi elaborado um fluxograma de forma a incluir na rotina do serviço de farmácia as atividades clínicas a serem desenvolvidas. Para isso foram considerados processos que compreendem a obtenção de dados clínicos dos pacientes, avaliação de possíveis problemas relacionados a medicamentos e, caso necessário, a realização de intervenções farmacêuticas.

Após elucidar o fluxograma das atividades, elaborou-se formulário visando facilitar e padronizar a realização do acompanhamento farmacoterapêutico dos pacientes. Neste continham informações sobre a identificação do paciente, histórico clínico e dados sobre a evolução clínica, parâmetros clínicos, exames laboratoriais, farmacoterapia atual e avaliação farmacoterapêutica, abrangendo informações acerca de interações medicamentosas, incompatibilidades por sonda e incompatibilidades físico-químicas.

Para definir quais parâmetros seriam utilizados no formulário de acompanhamento farmacoterapêutico, foi realizada uma ampla pesquisa bibliográfica sobre farmácia clínica buscando os principais parâmetros acompanhados pelo farmacêutico clínico e posteriormente cada parâmetro que se julgou necessário foi pesquisado particularmente para se justificar. Além disso, foi realizada também a consulta de outros formulários de acompanhamento clínico da própria instituição e de outros serviços de farmácia clínica. A pesquisa bibliográfica foi realizada utilizando os bancos de dados Portal de Periódicos Capes, SciELO e Pubmed, bem como trabalhos de conclusão de curso, dissertações de mestrado, teses de doutorado, livros, documentos governamentais e de instituições especializadas no tema. Foram utilizados os seguintes descritores para a pesquisa bibliográfica: farmácia clínica, acompanhamento farmacoterapêutico, identificação do paciente, histórico clínico, parâmetros clínicos, exames laboratoriais, farmacoterapia, avaliação farmacoterapêutica, intervenção farmacêutica, problemas relacionados a medicamentos, segurança do paciente.

Foram elaborados dois check-list para conferência dos itens que devem compor a prescrição e dos itens que devem ser analisados no acompanhamento farmacoterapêutico do paciente. O check-list da prescrição foi elaborado de acordo com o protocolo integrante do Programa Nacional de Segurança do Paciente sobre segurança na prescrição, uso e administração de medicamentos ${ }^{19}$. O check-list do acompanhamento farmacoterapêutico foi elaborado a fim de facilitar a conferência do processo a ser realizado pelo farmacêutico durante o acompanhamento diário do paciente, sendo esse elaborado com base no fluxograma de atividades clínicas.

Considerou-se também a elaboração um modelo de documento para registro em prontuário das intervenções farmacêuticas a serem realizadas, constando informações acerca das interações e/ou incompatibilidades medicamentosas observadas e suas respectivas condutas sugeridas. Este docu- mento, antes de ser anexado ao prontuário do paciente, deverá ser avaliado pelo médico e/ou enfermeiro responsável para validar a intervenção a ser realizada.

Por fim, pensando em proporcionar um controle das atividades clínicas desenvolvidas e facilitar futuras análises do serviço de farmácia clínica, foi elaborado um banco de dados para registro das intervenções farmacêuticas.

\section{RESULTADOS}

Inicialmente foi elaborado um fluxograma de atividades, abrangendo as atividades realizadas pelo serviço de farmácia (Figura 1) constando as atividades de separação, conferência e administração dos medicamentos prescritos, que são etapas cruciais para garantir a segurança e uso correto dos medicamentos. Com base nas atividades clínicas que o farmacêutico pode desenvolver ${ }^{5}$, foram selecionadas para serem implementadas inicialmente no serviço de farmácia clínica, incluídas no fluxograma, as seguintes atividades:

- Recebimento, triagem e análise criteriosa da prescrição, buscando possíveis erros de redação, transcrição, aprazamento e conciliação;

- Avaliação de possíveis problemas relacionados a medicamentos, envolvendo as intervenções farmacêuticas quando necessário;

- Consulta ao prontuário e avaliação clínico-laboratorial, visando à obtenção de dados clínicos importantes para o acompanhamento farmacoterapêutico dos pacientes;

- Registro em prontuário das intervenções farmacêuticas realizadas; e,

- Participação das discussões de casos clínicos, fornecendo informações a respeito do acompanhamento farmacoterapêutico dos pacientes e coletando informações referentes à evolução clínica do paciente.

O formulário de acompanhamento farmacoterapêutico busca facilitar o acompanhamento diário dos pacientes. Nele são registradas informações importantes sobre a evolução clínica do paciente, como por exemplo avaliação da farmacoterapia, progressão 
Figura 1. Fluxograma de atividades do Serviço de Farmácia Clínica na UTI Adulto, Rio de Janeiro, 2020.

\section{Fluxograma de Atividades do Serviço de Farmácia}

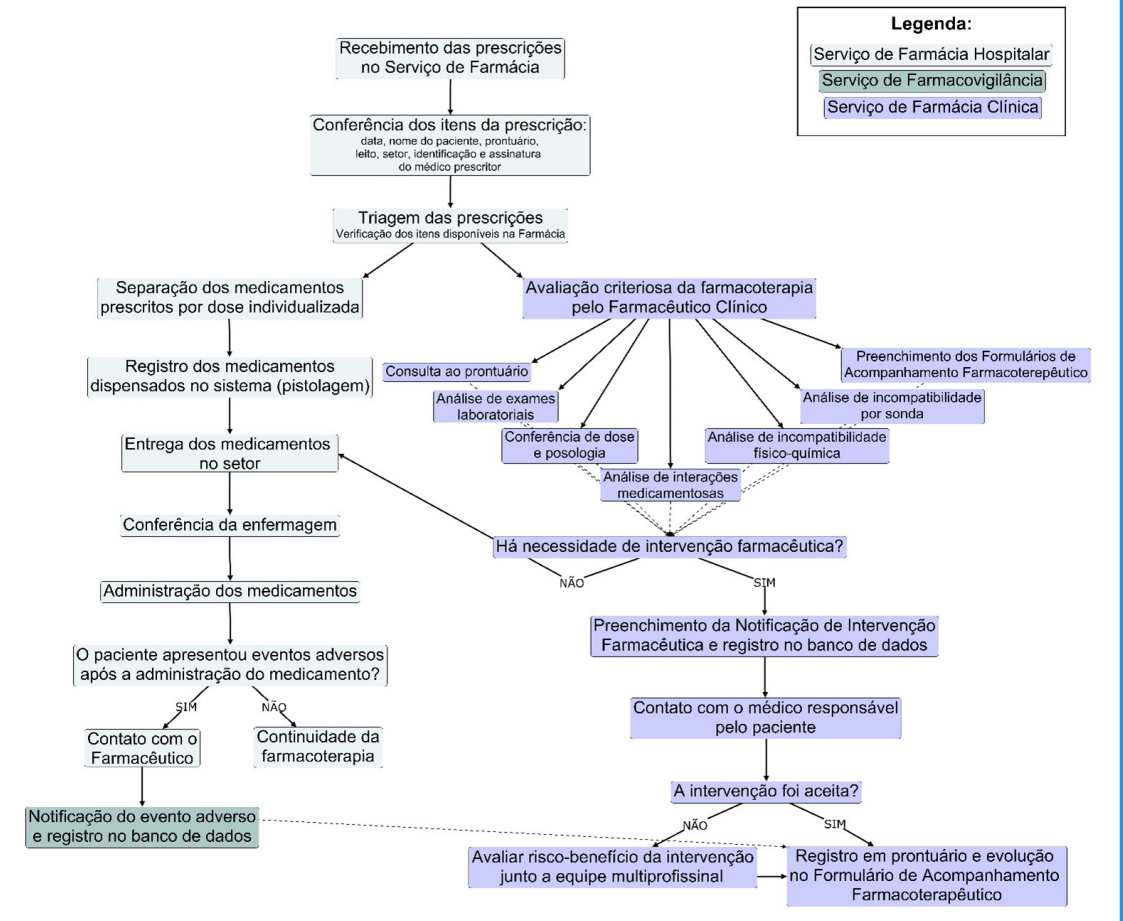

Fonte: elaboração própria, 2020

Figura 2. Formulário de acompanhamento farmacoterapêutico para pacientes críticos em UTI, Rio de Janeiro, 2020.

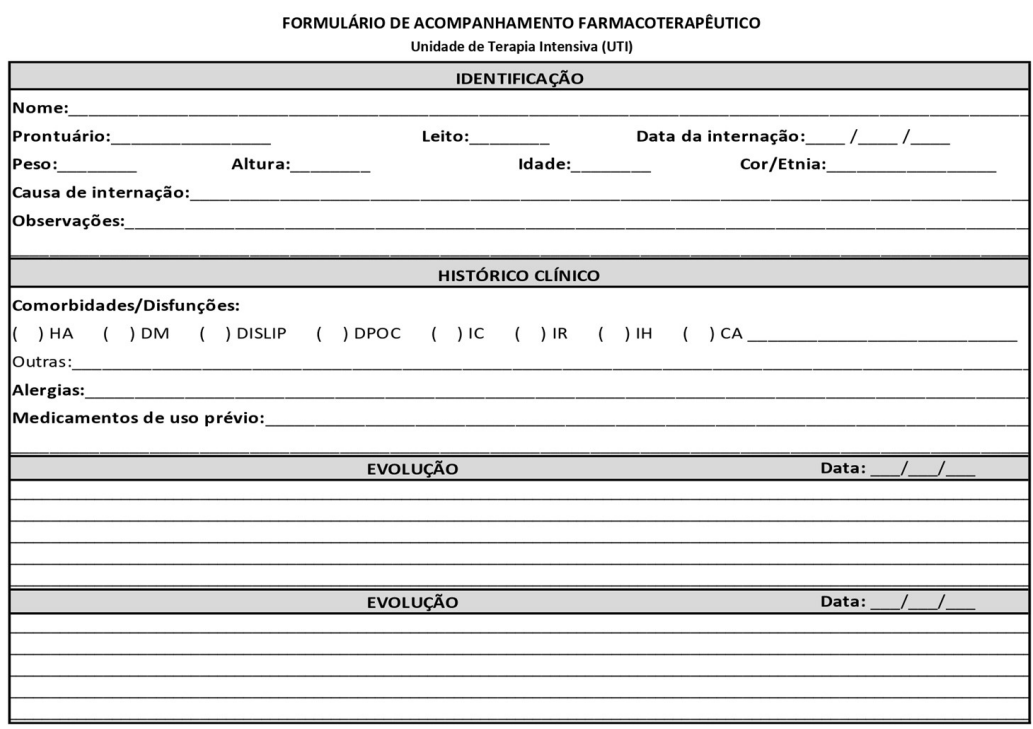

HA: hipertensão arterial; DM: diabetes mellitus; DISLIP: dislipidemias; DPOC: doença pulmonar obstrutiva crônica; IC: insuficiência cardíaca; IR: insuficiência renal; IH: insuficiência hepática; CA: câncer. ou regressão de infecções, problemas ativos, alteração de condutas médicas, ocorrência de reaçôes adversas, parâmetros clínicos alterados que necessitam de monitoramento mais rigoroso, entre outras informações. Esse formulário é dividido em identificação, evolução e acompanhamento farmacoterapêutico, foi elaborado pelas autoras e a partir da sua aprovação pela equipe foi implementado na rotina do serviço de farmácia clínica. (Figura 2).

$\mathrm{Na}$ identificação consta informações sobre características físicas do paciente (peso, altura, idade e cor/etnia) e causas da internação (data e causa de internação). Além disso, o formulário contém informações sobre histórico clínico do paciente (comorbidades e disfunções, alergias e medicamentos de uso prévio). O campo de evolução conta com registro da evolução clínica do paciente observada pelo farmacêutico (problemas ativos; dieta; uso de sonda e acessos venosos e/ou arteriais; em diálise; última evacuação). Sobre exames laboratriais, essenciais para garantir a individualização da terapia, estão: função renal, hepática e alterações hemodinâmicas. Sobre as informações acerca da farmacoterapia do paciente (medicamentos prescritos para o paciente) foi determinada com base da mnemônica FASTHUG-MAIDENS ${ }^{20}$.

$\mathrm{Na}$ avaliação farmacoterapêutica é registrada alterações realizadas na prescrição do paciente durante o dia (normalmente ao longo do round) e conduta farmacêutica (interaçôes medicamentosas, incompatibilidades por sonda e/ou físico-químicas). Foi elaborado também um formulário eletrônico de acompanhamento farmacoterapêutico de mesmo conteúdo do ilustrado na Figura 2, para manter registro eletrônico e facilitar a análise de alguns itens (conferência das doses dos medicamentos prescritos com as doses preconizadas em bula e em estudos clínicos).

O check-list da prescrição (Figura 3), elaborado de acordo com o protocolo integrante do Programa Nacional de Segurança do Paciente (PNSP) ${ }^{19}$ e do acompanhamento farmacoterapêutico contém itens analisados durante o acompanhamento, visando facilitar a conferência do farmacêutico quanto aos processos.

Foi elaborado um modelo para registro em prontuário das intervenções farmacêu- 


\section{artigo}

Christiani, R.S.; Rocha, G.C.; Nogueira, T.A.

Estruturação documental do serviço de farmácia clínica em uma unidade de terapia intensiva de um hospital público de grande porte

Figura 2. Continuação do Formulário de acompanhamento farmacoterapêutico para pacientes críticos em UTI, Rio de Janeiro, 2020.

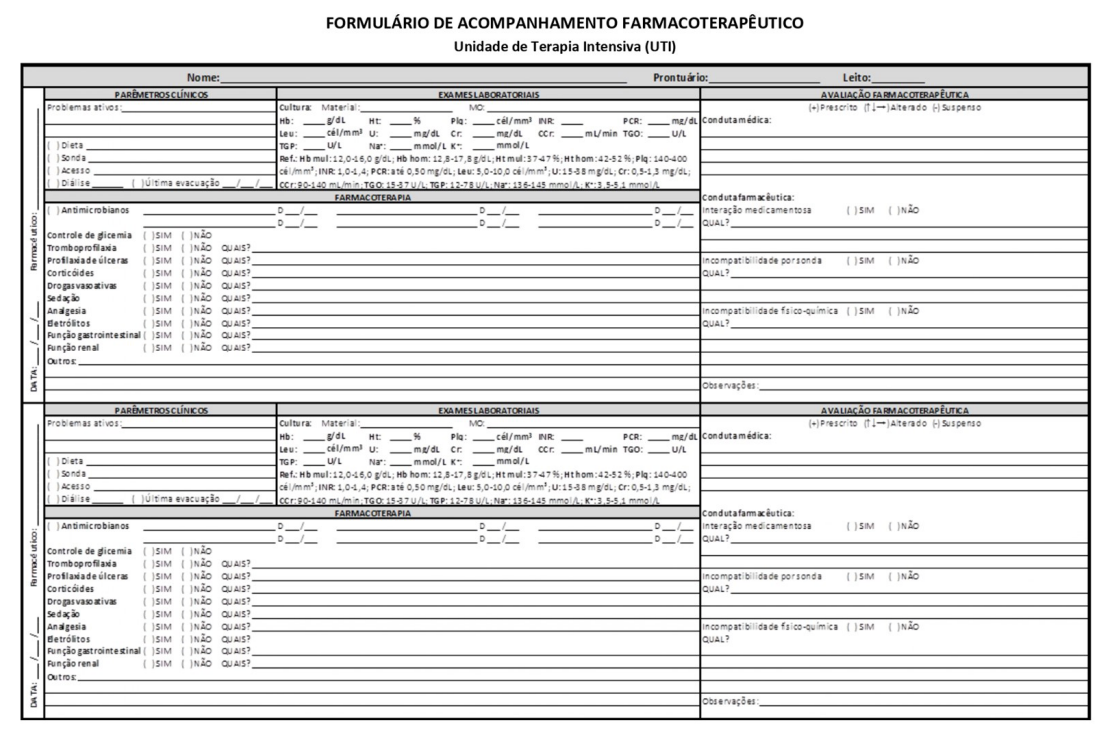

Hb: hemoglobina; Ht: hematócrito; Plq: plaquetas; INR: razão normalizada internacional; PCR: proteína C reativa; Leu: leucócitos; U: ureia; Cr: creatinina; CCr: clearance de creatinina; TGO: transaminase glutâmico-oxalacética; TGP: transaminase glutâmico-pirúvica; $\mathrm{Na}+$ : sódio; K+: potássio; MO: microrganismo.

Figura 3. Check-list da prescrição e check list de acompanhamento farmacoterapêutico para pacientes em centro de tratamento intensivo

\section{Check-list da prescriçăo}

\begin{tabular}{|l|l|}
\hline Identificação do paciente & \\
\hline Nome completo & \\
\hline Número do prontuário & \\
\hline Leito & \\
\hline Serviço & \\
\hline Nome do hospital & \\
\hline Identificação do prescritor & \\
\hline Nome completo & \\
\hline Número do CRM & \\
\hline Assinatura & \\
\hline Data & \\
\hline Legibilidade & \\
\hline Uso de abreviaturas & \\
\hline
\end{tabular}

ticas realizadas (Figura 4). A notificação de intervenção farmacêutica consta informações de identificação do paciente, o tipo de intervenção realizada, descrição do problema relacionado a medicamento (PRM) observado e descriçao da conduta sugerida. Além disso, avaliação da intervenção, onde o médico ou o enfermeiro poderá comunicar conduta. A partir das intervenções criou-se

\begin{tabular}{|l|l|}
\multicolumn{2}{|c|}{$\begin{array}{c}\text { Check-list de acompanhamento } \\
\text { farmacoterapêutico }\end{array}$} \\
\hline Conferência de doses & \\
\hline Exames Laboratoriais & \\
\hline Interações Medicamentosas & \\
\hline Incompatibilidades por sonda & \\
\hline Incompatibilidades físico-químicas & \\
\hline Ajuste de função renal & \\
\hline Ajuste de função hepática & \\
\hline Aprazamento & \\
\hline Controle de antimicrobianos & \\
\hline
\end{tabular}

um banco de dados (data, identificação do paciente, número da intervenção, tipo de intervenção, PRM, conduta farmacêutica, avaliação) para avaliação e acompanhamento com indicadores para controle do processo.

\section{DISCUSSÃO}

Segundo PNSP acerca da identificação correta dos pacientes, deve-se utilizar no mínimo dois identificadores (nome completo do paciente, nome completo da mãe, data de nascimento e/ou número de prontuário) ${ }^{21}$. Sendo assim, escolheu-se para registro no formulário de acompanhamento farmacoterapêutico o nome completo do paciente e o número de prontuário, bem como o número do leito ocupado (não confiável por ser mutável ao longo do processo), porém auxiliou na organização e separação dos formulários.

Características físicas (peso, altura, idade e cor/etnia) foram importantes para definir alguns parâmetros da farmacoterapia. $\mathrm{O}$ peso e a altura, auxiliou a determinação da superfície corpórea, com definição da dose terapêutica, especialmente os quimioterápicos ${ }^{22}$. A falha na aferição e regirto deste parâmetros pode implicar na ocorrência de erros de medicação graves e até fatais, especialmente se relacionados à medicamentos potencialmente perigosos, como anticoagulantes e quimioterápicos, sobretudo em pacientes idosos e pediátricos ${ }^{23}$.

$\mathrm{Na}$ avaliação da farmacoterapia conhecer o histórico prévio do paciente (comorbidades, disfunções e medicamentos em uso prévio) é fundamental, para possibilitar a conciliação medicamentosa. Segundo Lombardi e colaboradores (2016), até 27\% dos erros de prescrição podem estar relacionados a históricos de medicações incompletos, resultando em discrepâncias entre os medicamentos de uso antes da admissão e aqueles utilizados durante a internação ${ }^{24}$.

Sendo assim, a coleta adequada do histórico no momento da admissão é importante para garantir a segurança do paciente. Além disso, é importante conhecer o histórico de alergia do paciente, visto que aproximadamente de $25 \%$ a $30 \%$ das reações adversas a medicamentos são devidas a reações alérgicas e pseudo-alérgicas ${ }^{25,26}$.

A interação do fármaco com os alimentos ou componentes da dieta pode acarretar a redução da biodisponibilidade, podendo levar inclusive o desenvolvimento de efeitos adver$\operatorname{sos}{ }^{27,28}$. Um estudo multicêntrico realizado por Reis e colaboradores (2014) em UTI de sete hospitais de ensino no Brasil mostrou que dos 320 pacientes com 24 horas de internação em uso de nutrição enteral, 20 pacientes (6,3\%) apresentaram interações fármaco-nu- 
trição ${ }^{29}$. Outra preocupação é adsorção nas paredes da sonda enteral ${ }^{27,30}$.

A administração incorreta de medicamentos por sondas nasogástricas e nasoenteral pode acarretar em perda da sonda enteral ou ainda uma falha terapêutica, podendo gerar danos ao paciente ${ }^{30,31,32}$. Além disso, a instalação de uma nova sonda pode gerar problemas como desconforto do paciente, risco de posicionamento errado da sonda, custos adicionais de materiais e exames radiológicos para confirmação do posicionamento da sonda ${ }^{31}$.
Sendo assim, a presença de sondas nasogástricas e nasoenteral e sua compatibilidade com a farmacoterapia do paciente é um ponto de avaliação pelo farmacêutico.

Sobre acessos venosos e arteriais presentes no paciente, avalia-se incompatibilidades físico-químicas, bem como a análise do melhor acesso a ser utilizado na administração de cada medicamento ${ }^{33,34}$. A administração de medicamentos incompatíveis pode resultar em diversas alterações nos medicamentos administrados, levando ao comprometimen-
Figura 5. Modelo de notificação de intervenção farmacêutica em prontuário para pacientes em UTI, Rio de Janeiro, 2020.

NOTIFICAÇÃO DE INTERVENÇÃO FARMACÊUTICA Unidade de Terapia Intensiva (UTI)

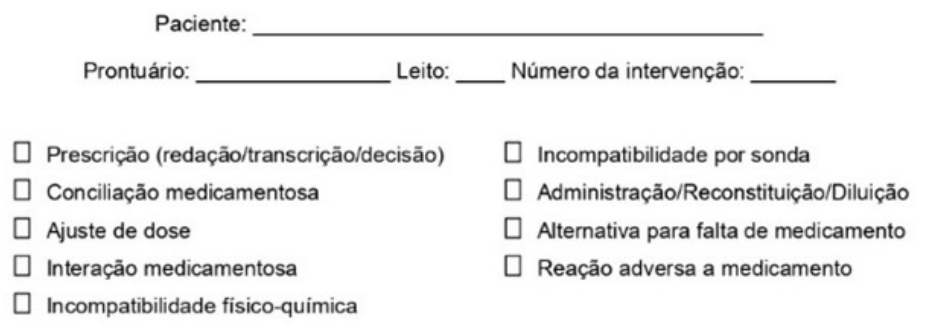

Problema relacionado a medicamento observado:

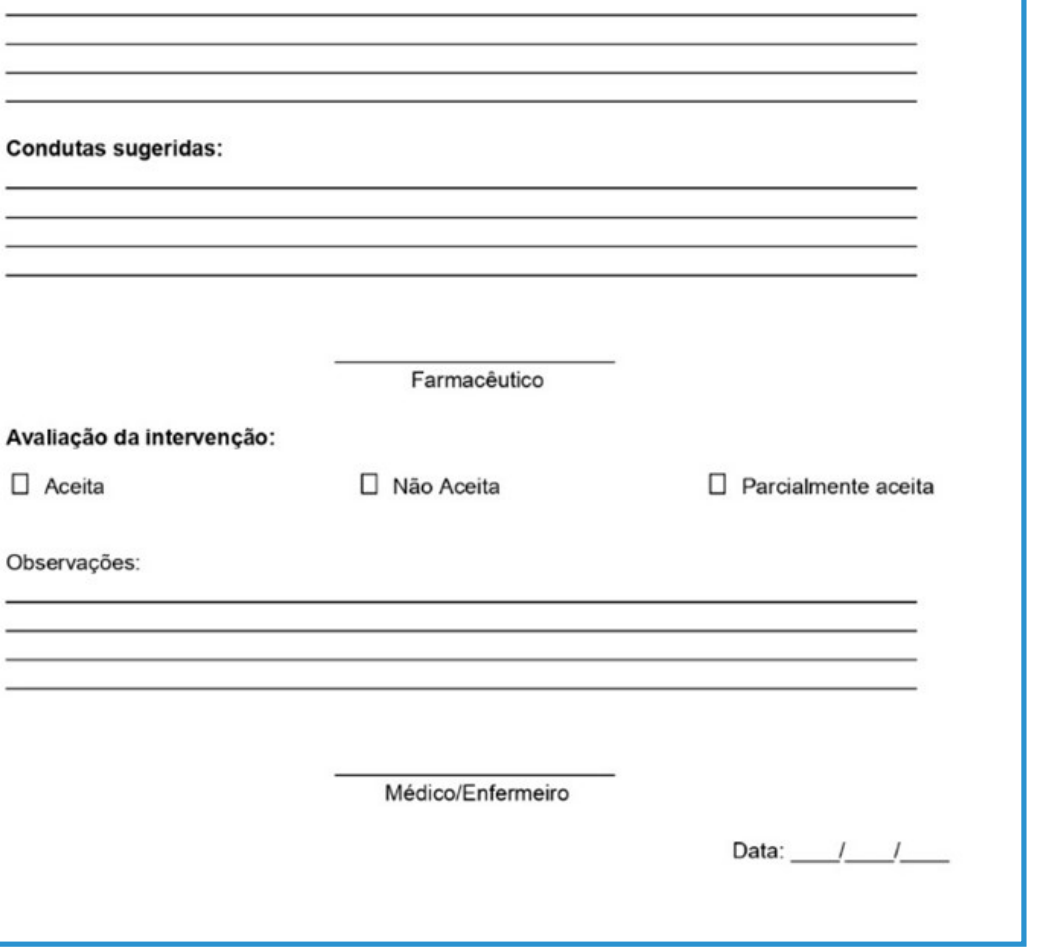

to da terapia e segurança do paciente. As alterações observadas incompatibilidade física são: precipitação, alterações de cor, liberação de gás e turvação. Enquanto incompatibilidade química são: diminuição de dose, degradação do medicamento, formação de produtos inativo ou tóxicos ${ }^{33}$.

Ressalta-se a influência da diálise (possibilidade de redução de níveis plasmáticos do fármaco), considera-se os rins essenciais na manutenção da homeostase do organismo, devido as suas funções regulatórias, excretórias e endócrinas ${ }^{35}$. Um estudo realizado em 2014 por Marquito e colaboradores concluiu que associação de medicamentos em indivíduos com doença renal crônica estava relacionada com alta prevalência de interações medicamentosas graves, com aumento da probabilidade de ocorrência em 2,5 vezes para cada medicamento adicional ${ }^{35}$. Assim, pacientes com comprometimento renal necessitam de ajuste de dose, destacando importância do monitoramento da farmacoterapia.

A constipação como efeito adverso frequente no uso de alguns medicamentos, tambpem exige ajustes a farmacoterapia ${ }^{36}$. Segundo Agra e colaboradores (2013) a prevalência estimada de constipação intestinal varia de $50 \%$ e $90 \%$, sendo mais elevada entre os pacientes em uso de analgésicos, sobretudo opioides $^{37}$. Porém anti-inflamatórios não esteroidais, anti-hipertensivos e antiarrítmicos bloqueadores do canal de cálcio, diuréticos, medicamentos que atuam no sistema nervoso (anticolinérgicos, antidepressivos tricíclicos, anticonvulsivantes, antiparkinsonianos, antipsicóticos, sedativos), entre outros medicamentos tambpem podem apresenta tal evento ${ }^{36}$.

Além disso, o farmacêutico deve estar atento ao monitoramento dos exames laboratoriais e, quando disponíveis kits diagnósticos, também ao monitoramento dos níveis séricos de fármacos, visando garantir a segurança e eficácia no uso de medicamentos, bem como propor, quando necessário, alterações para adequação da farmacoterapia ${ }^{38,39,40,41,42}$.

Para facilitar o registro e a avaliação do farmacêutico, a parte do formulário que abrange a farmacoterapia do paciente foi dividida em classes farmacológicas, baseada no FASTHUG-MAIDENS. Em 2005, Vincent propôs uma mnemônica FASTHUG 


\section{artigo}

como uma abordagem padronizada para auxiliar os médicos de UTI no atendimento aos pacientes críticos ${ }^{43}$. Em 2011, Mabasa e colaboradores modificaram a mnemônica para FASTHUT-MAIDENS, para que fosse possível identificar os problemas relacionados a medicamentos em UTI ${ }^{20}$.

Baseado nesta metodologia, as classes farmacológicas utilizadas no formulário foram: controle de glicemia, tromboprofilaxia, profilaxia de úlceras, sedação, analgesia, eletrólitos, antimicrobianos com controle de data de início e término do tratamento. Além disso, foram adicionadas algumas classes que possuíam prescrição recorrente, para facilitar o registro, sendo elas: corticoides, drogas vasoativas, função gastrointestinal e função renal.

A documentação/registro é uma etapa crucial para integração da assistência e a progressão do cuidado do paciente, é importante que essa seja eficaz e eficiente entre os profissionais de saúde ${ }^{10}$. Para isso, o farmacêutico deve documentar os cuidados prestados e as intervenções realizadas idealmente no prontuário, uma vez que esse documento é de acesso comum a todos os profissionais de saúde envolvidos no cuidado do paciente ${ }^{3,11}$.

Segundo as resoluções do CFF $n^{\circ} 585$ (2013) e 675 (2019), é atribuição clínica do farmacêutico fazer a evolução farmacêutica e registrar no prontuário do paciente para garantir a transição e a continuidade do cuidado $^{5,12}$. Ressalta-se importância do registro frequente e adequado, a fim possibilitar a continuidade das informações e promover um cuidado mais seguro ${ }^{44}$. Contudo, em constância deve-se assumir a avaliação do processo e adaptação da documentação sempre que oportuno, garantindo qualidade e assertividade do serviç̧o ${ }^{45,46}$.

\section{CONCLUSÃO}

O presente estudo possibilitou a criação de modelos de formulários a serem utilizados na prática clínica farmacêutica, com a finalidade de facilitar o acompanhamento farmacoterapêutico dos pacientes críticos e melhorar a qualidade do cuidado prestado.

A estruturação de um serviço de farmácia clínica é sempre complexa e deve ser bem estudada e elaborada especificamente para o grupo de paciente que visa acompanhar. Embora muitos serviços clínicos possam apresentar semelhanças, cada serviço possui sua peculiaridade, o que dificulta a criação de um modelo único de assistência.

Este trabalho tem por função otimizar a criação de documentos em outros serviços e justificar as informações pertinentes a prática clínica.

\section{REFERENCES}

1. Carneiro MB, Souza AR, Cambrussi MC, Cremonini MC, Ferreira VC. Da teoria a prática: experiência de implantação das atividades de Farmácia Clínica em Hospital Oncológico [Internet]. Curitiba: CFF; 2011 [cited 2020 jun 12]. Available from: https:// www.cff.org.br/userfiles/2011\%20-\%20Segundo\%20Lugar $\% 20$ -\%20Marcela\%20 Bechara\%20-\%20Da\%20teoria\%20a\%20pratica\%20Farmacia\%20Clinica\%20no\%20 HEG(1).pdf

2. Costa JM, Abelha LL, Duque FA. Experiência de implantação do serviço de farmácia clínica em um hospital de ensino. Rev. Bras. Farm.2013; 94 (3): 250-256.

3. Ferracini FT, Almeida SM, Locatelli J, Petriccione S, Haga CS. Implantação e evolução da farmácia clínica no uso racional de medicamentos em hospital terciário de grande porte. Einstein. 2011; 9(4 Pt 1): 456-60.

4. Magalhães AC, Cantanhede AM, Drummond BM, Drumond, YA, Miranda VF. Avaliação da implantação do serviço de farmácia clínica na Unidade de Terapia Intensiva para contribuir na segurança do paciente. Rev Med Minas Gerais. 2016; 26 (Supl 5): S16-S22.

5. Brasil. Resolução n. 585, de 29 de agosto de 2013. Regulamenta as atribuições clínicas do farmacêutico e dá outras providências. Diário Oficial da União, Brasília, DF, n.186, p. 186188, 25 set. 2013, Seção 1.

6. Miranda TM, Petriccione S, Ferracini FT, Borges WM Filho. Intervenções realizadas pelo farmacêutico clínico na unidade de primeiro atendimento. Einstein. 2012; 10(1):74-8.

7. Fernandes HS, Pulzi SA Júnior, Costa RC Filho. Qualidade em terapia intensiva. Rev Bras Clin Med. 2010; 8:37-45.

8. Holand RW, Nimmo CM. Transitions, part 1: Beyond pharma- ceutical care. Am J Health-Syst Pharm. 1999; 56(1): 1758-1764.

9. Okumura LM, Silva DM, Comarella L. Relação entre o uso seguro de medicamentos e Serviços de Farmácia Clínica em Unidades de Cuidados Intensivos Pediátricos. Rev Paul Pediatr. 2016; 34(4): 397-402.

10. ASHP. Guidelines on Documenting Pharmaceutical Care in Patient Medical Records. Am J Health-Syst Pharm. 2003; 60:705-7.

11. Araújo PS, Costa EA, Guerra AA Junior. Atividades farmacêuticas de natureza clínica na atenção básica no Brasil. Rev Saude Publica. 2017; 51 Supl 2:6s.

12. Brasil. Resolução n. ${ }^{\circ} 675$, de 31 de outubro de 2019. Regulamenta as atribuições do farmacêutico clínico em unidades de terapia intensiva, e dá outras providências. Diário Oficial da União, Brasília, DF, n.225, p.128, 31 out. 2019, Seção 1.

13. Araújo RQ, Almeida SM. Farmácia clínica na Unidade de Terapia Intensiva. Rev Pharmacia Brasileira. 2008; 1-4.

14. Pilau R, Hegele V, Heineck I. Atuação do farmacêutico clínico em unidade de terapia intensiva adulto: uma revisão da literatura. Rev. Bras. Farm. Hosp. Serv. Saúde São Paulo. 2014 jan./ mar.; 5(1): 19-24.

15. Fideles GMA, Alcântara JM Neto, Peixoto AA Júnior, Souza PJ Neto, Tonete $T L$, Silva JE, et al. Recomendações farmacêuticas em unidade de terapia intensiva: três anos de atividades clínicas. Rev Bras Ter Intensiva. 2015; 27(2): 149-154.

16. Brilli RJ, Spevetz A, Branson RD, Campbell GM, Cohen H, Dasta JF, et al. Critical care delivery in the intensive care unit: defining clinical roles and the best practice model. Crit Care Med. 2001 


\section{REFERENCES}

Oct; 29(10): 2007-2019.

17. SCCM e ACCP. Position paper on critical care pharmacy services. Society of Critical Care Medicine and American College of Clinical Pharmacy Task Force on Critical Care Pharmacy Services. Crit Care Med. 2000 Nov.; 28(11): 3746-3750.

18. Pereira GA Júnior, Coletto FA, Martins MA, Marson F, Pagnano RC, Dalri MC, et al. 0 papel da unidade de terapia intensiva no manejo do trauma. Medicina, Ribeirão Preto. 1999; 32: 419-437.

19. Brasil. Ministério da Saúde. Documento de referência para o Programa Nacional de Segurança do Paciente. Anexo 03: Protocolo de segurança na prescrição, uso e administração de medicamentos. Ministério da Saúde; Fundação Oswaldo Cruz; Agência Nacional de Vigilância Sanitária. Brasília: Ministério da Saúde, 2014.

20. Mabasa VH, Malyuk DL, Weatherby EM, Chan A. A Standardized, Structured Approach to Identifying Drug-Related Problems in the Intensive Care Unit: FASTHUG-MAIDENS. Can J Hosp Pharm. 2011 Sep./Oct.; 64(5): 366-369.

21. Brasil. Ministério da Saúde. Documento de referência para o Programa Nacional de Segurança do Paciente. Anexo 02: Protocolo de identificação do paciente. Ministério da Saúde; Fundação Oswaldo Cruz; Agência Nacional de Vigilância Sanitária. Brasília: Ministério da Saúde, 2014.

22. Ribeiro TS, Santos VO. Segurança do Paciente na Administração de Quimioterapia Antineoplásica: uma Revisão Integrativa. Rev Bras Cancerol. 2015; 61(2): 145-153.

23. ISMP. Weight-Based Medication Dose Errors. ISMP Canada Safety Bulletin. 2016; 16(9).

24. Lombardi NF, Mendes AE, Lucchetta RC, Reis WC, Fávero ML, Correr CJ. Análise das discrepâncias encontradas durante a conciliação medicamentosa na admissão de pacientes em unidades de cardiologia: um estudo descritivo. Rev Latino-Am Enfermagem. 2016; 24: e2760.

25. Bernd LA. Alergia a Medicamentos. Rev Bras Alerg Imunopatol. 2005; 28(3): 125-132.

26. Ensina LF, Fernandes FR, Gesu GD, Malaman MF, Chavarria ML, Berned LA. Reações de hipersensibilidade a medicamentos. Rev Bras Alerg Imunopatol. 2009; 32(2): 178-183.

27. Reis AM. Fatores associados às interações medicamentosas potenciais e aos eventos adversos a medicamentos em uma unidade de terapia intensiva [Tese de doutorado]. Escola de Enfermagem de Universidade de São Paulo, Ribeirão Preto, 2009.

28. Heldt T, Loss SH. Interação fármaco-nutriente em unidade de terapia intensiva: revisão da literatura e recomendações atuais. Rev Bras Ter Intensiva. 2013; 25(2): 162-167.

29. Reis AM, Carvalho RE, Faria LM, Oliveira RC, Zago KS, Cavelagna MF, et al. Prevalência e significância clínica de interações fármaco-nutrição enteral em Unidades de Terapia Intensiva. Rev Bras Enferm. 2014 jan-fev; 67(1): 85-90.

30. CFF. Administração de medicamentos por sonda. Boletim Farmacoterapêutica. Ano XIV, Números 03 e 04, mai-ago, 2009.
31. Lima G, Negrini NM. Assistência farmacêutica na administração de medicamentos via sonda: escolha da forma farmacêutica adequada. Einstein. 2009; 7(1 Pt 1): 9-17.

32. Catalán E, Padilla F, Hervás F, Pérez MA, Ruiz F. Fármacos orales que no deben ser triturados. Enferm Intensiv. 2001; 12(3): 146-150.

33. Garcia JH. Incompatibilidade de medicamentos intravenosos e fatores de risco em pacientes críticos: coorte histórico [Dissertação de mestrado]. Escola de Enfermagem da Universidade de São Paulo, São Paulo, 2015.

34. Santos MT, Hegele V, Hoffmann TD, Chiarani F, Hennigen FW. Instrumento para avaliação da compatibilidade em $Y$ na administração intravenosa de medicamentos em Unidades de Terapia Intensiva. Rev Bras Farm Hosp Serv Saúde São Paulo. 2013 jul./ set.; 4(3): 34-37.

35. Marquito AB, Fernandes NM, Colugnati FA, Paula RB. Interações medicamentosas potenciais em pacientes com doença renal crônica. J Bras Nefrol 2014; 36(1): 26-34.

36. Andrade MA, Silva MV, Mendonça S, Freitas O. Assistência farmacêutica frente à obstipação intestinal no idoso. Infarma. 2003 Set/Out.; 15(9-10): 64-69.

37. Agra G, Fernandes MA, Platel IC, Barros RC, Freire ME. Constipação em pacientes com doença oncológica avançada em uso de opioides. O Mundo da Saúde, São Paulo. 2013;37(4): 472-478.

38. CRF-SP. Farmácia Clínica. Comissão Assessora de Farmácia Clínica, Conselho Regional de Farmácia do Estado de São Paulo. $1^{\text {a }}$ edição-1ª reimpressão. 44 p. São Paulo, 2015.

39. Mauri MC, Paletta S, Di Pace, C, Reggiori A, Cirnigliaro G, Valli I, et al. Clinical Pharmacokinetics of Atypical Antipsychotics: An Update. Clin Pharmacokinet. 2018; 57(12): 1493-1528.

40. Chen G, Hojer AM, Areberg J, Nomikos G. Vortioxetine: Clinical Pharmacokinetics and Drug Interactions. Clin Pharmacokinet. 2018; 57: 673-686.

41. Altamura AC, Moro AR, Percudani M. Clinical Pharmacokinetics of Fluoxetine. Clin. Phannacokinet. 1994; 26(3): 201-214.

42. Scholz J, Steinfathl M, Schulz M. Clinical Pharmacokinetics of Alfentanil, Fentanyl and Sufentanil. Clin. Pharmacokinet. 1996 Oct; 31(4): 275-292.

43. Vincent JL. Give your patient a fast hug (at least) once a day. Crit Care Med. 2005; 33(6): 1225-1229.

44. Santos LM. Comunicação relacionada ao uso de medicamentos por enfermeiros, farmacêuticos e médicos: uma análise dos registros em prontuários clínicos [Trabalho de conclusão de curso de graduação]. Faculdade de Farmácia de Universidade Federal de Sergipe, São Cristóvão, 2017.

45. Al-Shaqha W, Zairi M. The role of quality in pharmacêutical care management. Managing Service Quality. 2001; 11(1): 32-39.

46. Cillis M, Spinewine A, Krug B, Quennery S, Wouters D, Dalleur 0 . Development of a tool for benchmarking of clinical pharmacy activities. Int I Clin Pharm. 2018; 40(6): 1462-1473. 